\title{
RIESZ BASIS GENERATION, EIGENVALUES DISTRIBUTION, AND EXPONENTIAL STABILITY FOR A EULER-BERNOULLI BEAM WITH JOINT FEEDBACK CONTROL
}

\author{
BAO-ZHU GUO and K.Y. CHAN
}

\begin{abstract}
Using an abstract result on Riesz basis generation for discrete operators in general Hilbert spaces, we show, in this article, that the generalized eigenfunctions of an Euler-Bernoulli beam equation with joint linear feedback control form a Riesz basis for the state space. The spectrum-determined growth condition is hence obtained. Meanwhile, the exponential stability as well as the asymptotic expansion of eigenvalues are also readily obtained by a straightforward computation.
\end{abstract}

\section{Introduction}

For a vibrating system, the most exciting property is that the generalized eigenfunctions of the system form a Riesz basis for the state Hilbert space. The Riesz basis generation will trivially conclude many other important properties such as the spectrum-determined growth condition that is significant both theoretically and practically but often not so easy to determine for infinite dimensional systems. Since the general model of serially connected Euler-Bernoulli beams with joint linear feedback control was proposed in Chen et al. (1987), many efforts have been made to study the asymptotic distribution of the eigenvalues (Chen et al. , 1989, 1990), and the exponential stability (Rebarber,1995) of the two connected-beam equations. However, the Riesz basis generation property and the spectrum-determined growth condition have not been

2000 Mathematics Subject Classification: 35P20, 93D15.

Servicio de Publicaciones. Universidad Complutense. Madrid, 2001 
reported until recently; the problem of a one beam equation was examined by Conrad and Morgul, (1998); Guo, (2001a). In Guo (2001a), an abstract result on the Riesz basis generation for discrete operators in general Hilbert spaces is presented, by which the feedback effect for beam equations can be considered as the perturbation of an appropriately chosen free Riesz system. The generalized eigenfunctions between the controlled system and the free system are compared to get the Riesz basis property by estimating only those "high eigenmodes". The success in the choice of the appropriate auxiliary Riesz system relies on the observation that the characteristic equation of the controlled system is a lower order perturbation of that of free Riesz system, which is usually true for serially connected beam equations with joints linear feedback control. This idea has been applied successfully to get Riesz basis generation property for other systems such as the one dimensional linear thermoelastic equation (Guo, 2001b).

In this paper, we shall use this abstract result to prove that there is a sequence of generalized eigenfunctions of two connected Euler-Bernoulli beam equations with joint linear feedback control, which forms a Riesz basis for the state Hilbert space. The spectrum-determined growth condition is thus concluded from the algebraic simplicity of eigenvalues. Meanwhile, as a consequence, an asymptotic expansion of the eigenvalues as well as the exponential stability are readily obtained by direct symbolic computation, which greatly improves and simplifies similar results developed by asymptotic techniques for eigenvalues (Chen et al. , 1989, 1990) and the frequency domain approach for exponential stability (Rebarber, 1995).

\section{The explicit expression of eigenfunctions}

Since the game is almost the same for other types of boundary conditions and joint linear feedback controls, we demonstrate the whole process by considering the following Euler-Bernoulli beam equation with the simply 
supported end conditions and shear stabilizer at the joint $d, 0<d<1$ :

$$
\left\{\begin{array}{l}
y_{t t}(x, t)+y_{x x x x}(x, t)=0,0<x<d, d<x<1 \\
y(0, t)=y_{x x}(0, t)=0, y(1, t)=y_{x x}(1, t)=0, \\
y\left(d^{+}, t\right)=y\left(d^{-}, t\right), y_{x}\left(d^{+}, t\right)=y_{x}\left(d^{-}, t\right), y_{x x}\left(d^{+}, t\right)=y_{x x}\left(d^{-}, t\right), \\
y_{x x x}\left(d^{-}, t\right)-y_{x x x}\left(d^{+}, t\right)=K y_{t}(d, t)
\end{array}\right.
$$

where $K$ is a real constant. To make system (1) into a framework of semigroups, we introduce the underlying state Hilbert space $\mathcal{H}=$ $H_{E}^{2}(0,1) \times L^{2}(0,1)$, with the inner product induced norm:

$$
\|(f, g)\|^{2}=\int_{0}^{1}\left[\left|f^{\prime \prime}(x)\right|^{2}+|g(x)|^{2}\right] d x
$$

where $H_{E}^{2}(0,1)=\left\{f \in H^{2}(0,1) \mid f(0)=f(1)=0\right\}$. System (1) is then written as an evolutionary equation in $\mathcal{H}$ :

$$
\frac{d}{d t} Y(t)=\mathcal{A} Y(t)
$$

where $Y(t)=\left(y(\bullet, t), y_{t}(\bullet, t)\right) \in \mathcal{H}$ and $\mathcal{A}$ is defined by

$$
\mathcal{A}(f, g)=\left(g(x),-f^{(4)}(x)\right)
$$

with

$$
\begin{aligned}
D(\mathcal{A})= & \left\{(f, g) \in H_{E}^{2}(0,1) \times H_{E}^{2}(0,1) \mid\right. \\
& \left.f\right|_{[0, d]} \in H^{4}(0, d),\left.f\right|_{[d, 1]} \in H^{4}(d, 1), \\
& f^{\prime \prime}\left(d^{-}\right)=f^{\prime \prime}\left(d^{+}\right), \\
& \left.f^{\prime \prime \prime}\left(d^{-}\right)-f^{\prime \prime \prime}\left(d^{+}\right)=K g(d), f^{\prime \prime}(0)=f^{\prime \prime}(1)=0\right\}
\end{aligned}
$$

where $\left.f\right|_{[a, b]}$ denotes the function $f$ confined to $[a, b]$.

The following lemma is straightforward.

Lemma 1. $\mathcal{A}^{-1}$ exists and is compact on $\mathcal{H}$. Therefore, $\sigma(\mathcal{A})$ consists of isolated eigenvalues only.

The task in the sequel of this section is to find an explicit expression for any eigenfunction of $\mathcal{A}$. It is easily seen that any eigenfunction of $\mathcal{A}$ corresponding to $\lambda \in \sigma(\mathcal{A})$ takes the form $(\phi, \lambda \phi) \in D(\mathcal{A})$, where $\phi$ satisfies

$$
\left\{\begin{array}{l}
\lambda^{2} \phi(x)+\phi^{(4)}(x)=0,0<x<d, d<x<1 \\
\phi(0)=\phi^{\prime \prime}(0)=\phi(1)=\phi^{\prime \prime}(1)=0 \\
\phi\left(d^{-}\right)=\phi\left(d^{+}\right), \phi^{\prime}\left(d^{-}\right)=\phi^{\prime}\left(d^{+}\right), \phi^{\prime \prime}\left(d^{-}\right)=\phi^{\prime \prime}\left(d^{+}\right) \\
\phi^{\prime \prime \prime}\left(d^{-}\right)-\phi^{\prime \prime \prime}\left(d^{+}\right)=K \lambda \phi(d)
\end{array}\right.
$$


The problem now is to find the nonzero solutions $\phi$ to equation (5). To this end, let $\lambda=i \tau^{2}$. First, the general solutions of

$$
\left\{\begin{array}{l}
\lambda^{2} \phi(x)+\phi^{(4)}(x)=0,0<x<d, \text { or } d<x<1 \\
\phi(0)=\phi^{\prime \prime}(0)=\phi(1)=\phi^{\prime \prime}(1)=0
\end{array}\right.
$$

are of the form

$$
\left\{\begin{array}{l}
\phi(x)=c_{1} \operatorname{sh} \tau x+c_{2} \sin \tau x, 0 \leq x \leq d, \\
\phi(x)=d_{1} \operatorname{sh} \tau(1-x)+d_{2} \sin \tau(1-x), d<x \leq 1
\end{array}\right.
$$

where $c_{i}, d_{i}, i=1,2$, are constants and $\operatorname{ch} \tau=\cosh \tau, \operatorname{sh} \tau=\sinh \tau$ denote hyperbolic functions throughout the paper. Next, the condition $\phi\left(d^{-}\right)=$ $\phi\left(d^{+}\right)$reads

$$
c_{1} \operatorname{sh} \tau d+c_{2} \sin \tau d=d_{1} \operatorname{sh} \tau(1-d)+d_{2} \sin \tau(1-d),
$$

while $\phi^{\prime \prime}\left(d^{-}\right)=\phi^{\prime \prime}\left(d^{+}\right)$yields

$$
c_{1} \operatorname{sh} \tau d-c_{2} \sin \tau d=d_{1} \operatorname{sh} \tau(1-d)-d_{2} \sin \tau(1-d) .
$$

Adding (8) and $\pm(9)$ separately gives

$$
c_{1} \operatorname{sh} \tau d=d_{1} \operatorname{sh} \tau(1-d), c_{2} \sin \tau d=d_{2} \sin \tau(1-d) .
$$

Hence

$$
c_{1}=\frac{A}{\Delta} d_{1}, c_{2}=\frac{B}{\Delta} d_{2}
$$

where

$$
\Delta=\operatorname{sh} \tau d \sin \tau d, A=\operatorname{sh} \tau(1-d) \sin \tau d, B=\operatorname{sh} \tau d \sin \tau(1-d) .
$$

Thirdly, substituting (11) into the condition $\phi^{\prime}\left(d^{-}\right)=\phi^{\prime}\left(d^{+}\right)$, we obtain

$$
\operatorname{ch} \tau d \frac{A}{\Delta} d_{1}+\cos \tau d \frac{B}{\Delta} d_{2}=-d_{1} \operatorname{ch} \tau(1-d)-d_{2} \cos \tau(1-d) .
$$

Hence

$$
d_{1}=-B \cos \tau d-\Delta \cos \tau(1-d), d_{2}=A \operatorname{ch} \tau d+\Delta \operatorname{ch} \tau(1-d) .
$$

Substituting (13) back into (11) reduces to

$$
c_{1}=-\frac{A B}{\Delta} \cos \tau d-A \cos \tau(1-d), c_{2}=\frac{A B}{\Delta} \operatorname{ch} \tau d+B \operatorname{ch} \tau(1-d) .
$$


Furthermore, substituting (12) into (13) and (14), after simple calculation, we find that

$$
\left\{\begin{array}{l}
c_{1}=-\operatorname{sh} \tau(1-d) \sin \tau \\
c_{2}=\operatorname{sh} \tau \sin \tau(1-d) ; \\
d_{1}=-\operatorname{sh} \tau d \sin \tau ; \\
d_{2}=\operatorname{sh} \tau \sin \tau(1-d) .
\end{array}\right.
$$

Finally substituting (15) into (7), we find the expression of $\phi$ :

$$
\phi(x)=\left\{\begin{array}{l}
-s h \tau(1-d) \sin \tau \operatorname{sh} \tau x+\operatorname{sh} \tau \sin \tau(1-d) \sin \tau x, x \in[0, d], \\
-\operatorname{sh} \tau d \sin \tau \operatorname{sh} \tau(1-x)+\operatorname{sh} \tau \sin \tau d \sin \tau(1-x), x \in[d, 1] .
\end{array}\right.
$$

Lemma 2. Suppose $\phi \neq 0$, where $\phi(x)$ is given by (16). Then $\phi$ is the unique linearly independent eigenfunction (up to a scalar) of $\mathcal{A}$ corresponding to $\lambda=i \tau^{2}$, where $\tau$ is the solution of the following characteristic equation

$$
2 \tau \operatorname{sh} \tau \sin \tau=K i[\operatorname{sh} \tau(1-d) \sin \tau \operatorname{sh} \tau d-\operatorname{sh} \tau \sin \tau(1-d) \sin \tau d] .
$$

Proof. We need only show the last part. It is seen from previous discussions that the function $\phi$ defined by (16) satisfies

$$
\left\{\begin{array}{l}
\lambda^{2} \phi(x)+\phi^{(4)}(x)=0,0<x<d, d<x<1, \\
\phi(0)=\phi^{\prime \prime}(0)=\phi(1)=\phi^{\prime \prime}(1)=0, \\
\phi\left(d^{+}\right)=\phi\left(d^{-}\right), \phi^{\prime}\left(d^{+}\right)=\phi^{\prime}\left(d^{-}\right), \phi^{\prime \prime}\left(d^{+}\right)=\phi^{\prime \prime}\left(d^{-}\right)
\end{array}\right.
$$

for any $\lambda=i \tau^{2}$. Suppose $\phi \neq 0$. Then for $\lambda=i \tau^{2}$ to be an eigenvalue of $\mathcal{A}$, it is necessary and sufficient that the last condition

$$
\phi^{\prime \prime \prime}\left(d^{-}\right)-\phi^{\prime \prime \prime}\left(d^{+}\right)=\mathcal{K} \lambda \phi(d)
$$

should be satisfied. From (16)

$$
\phi^{\prime \prime \prime}\left(d^{-}\right)-\phi^{\prime \prime \prime}\left(d^{+}\right)=-2 \tau^{3} \operatorname{sh} \tau \sin \tau
$$

and

$$
\phi(d)=-\operatorname{sh} \tau(1-d) \sin \tau \operatorname{sh} \tau d+\operatorname{sh} \tau \sin \tau(1-d) \sin \tau d .
$$

Substituting (20) and (21) into (19), we get the characteristic equation (17). The proof is complete. 


\section{The asymptotic expression of eigenvalues}

It should be noted that the characteristic equation (17) indicates the following facts:

(a). For $K=0$, (17) becomes $\operatorname{sh} \tau \sin \tau=0$ which is just the characteristic equation of the following free system:

$$
\left\{\begin{array}{l}
y_{t t}(x, t)+y_{x x x x}(x, t)=0,0<x<1 \\
y(0, t)=y_{x x}(0, t)=0, y(1, t)=y_{x x}(1, t)=0 .
\end{array}\right.
$$

The system operator $\mathcal{A}_{0}$ associated with (22) is nothing but the operator $\mathcal{A}$ with $K=0$

$$
\left\{\begin{array}{l}
\mathcal{A}_{0}(f, g)=\left(g(x),-f^{(4)}(x)\right) \\
D\left(\mathcal{A}_{0}\right)=\left\{(f, g) \in\left(H_{E}^{2} \cap H^{4}\right) \times H_{E}^{2} \mid f^{\prime \prime}(0)=f^{\prime \prime}(1)=0\right\} .
\end{array}\right.
$$

$\mathcal{A}_{0}$ is skew-adjoint with compact resolvent in $\mathcal{H}$. By general operator theory, system (22) is a Riesz system, namely, there is a set of the maximal $\omega$-linearly independent (i.e. the sequence itself is $\omega$-linearly independent and if any element is added to this sequence, the expanded sequence must not be $\omega$-linearly independent anymore) sequence of eigenfunctions of system (22) which forms a Riesz (orthogonal) basis for $\mathcal{H}$.

(b). As $\operatorname{Re} \tau \rightarrow \infty, \operatorname{Im}|\tau|$ bounded, and $e^{\gamma \operatorname{Re} \tau}$ grows faster than any polynomial of $\tau$ as $|\tau| \rightarrow \infty$ for any $\gamma>0$, the right hand side of (17) caused, by feedback from free system (22), is a perturbation of the left hand side with an order of $\mathcal{O}\left(|\tau|^{-1}\right)$ as $|\tau| \rightarrow \infty$.

(c). Because of facts (a) and (b), we may say roughly that the controlled system (1) is a lower order perturbation of the free system (22).

Keeping these facts in mind is very important because in the sequel, we need free system (22) to be a reference Riesz system. The choice of auxiliary free system is based on the principle expressed by these facts. For serially connected Euler-Bernoulli beams with joint linear feedback control, we can use this principle to find a suitable auxiliary free system.

Theorem 1. There is a family of eigenvalues $\lambda_{n}=\left\{i \tau_{n}^{2},-i \bar{\tau}_{n}^{2}\right\}$ of $\mathcal{A}$ for all sufficiently large positive integer $n$, satisfying the following asymptotic expression

$$
\lambda_{n}=i \tau_{n}^{2}=i(n \pi)^{2}-K \sin ^{2} n d \pi+\mathcal{O}\left(n^{-1}\right) .
$$


Proof. Consider (17) in a small neighborhood $\mathcal{U}_{n}$ of $n \pi:|\tau-n \pi| \leq C$, where $C>0$ is a small constant and $n$ is a positive integer. Note that

$$
\left|e^{-\tau} \operatorname{sh} \tau\right|,\left|e^{-\tau} \operatorname{ch} \tau\right|,|\sin \tau|,|\cos \tau|
$$

are uniformly bounded on all $\mathcal{U}_{n}$. Multiplying $2 \tau^{-1} e^{-\tau}$ on both sides of (17), we can write (17) to be

$$
\left\{\begin{array}{l}
\sin \tau=\mathcal{O}\left(|\tau|^{-1}\right) \text { or } \\
\sin \tau=\frac{K i}{2 \tau}[\sin \tau-\sin d \tau \sin (1-d) \tau]+\mathcal{O}\left(e^{-\gamma \operatorname{Re} \tau}\right)
\end{array}\right.
$$

for some $\gamma>0$, uniformly on all $\mathcal{U}_{n}$. For each sufficiently large $n$, applying Rouche's theorem to the first equation in (25) in a small circle contained in $\mathcal{U}_{n}$ and centered at $n \pi$, we find a solution $\tau_{n}$ of the first equation of $(25)$ to be the form

$$
\tau_{n}=n \pi+\mathcal{O}\left(n^{-1}\right) .
$$

Substituting (26) into the second equation of (25) yields

$$
\cos n \pi \mathcal{O}\left(n^{-1}\right)=\frac{K i}{2 n \pi} \cos n \pi \sin ^{2} n d \pi+\mathcal{O}\left(n^{-2}\right) .
$$

Comparing the order of both sides above gives

$$
\mathcal{O}\left(n^{-1}\right)=\frac{K i}{2 n \pi} \sin ^{2} n d \pi+\mathcal{O}\left(n^{-2}\right)
$$

and so $\tau_{n}=n \pi+\frac{K i}{2 n \pi} \sin ^{2} n d \pi+\mathcal{O}\left(n^{-2}\right)$. Therefore,

$$
\lambda_{n}=i \tau_{n}^{2}=i(n \pi)^{2}-K \sin ^{2} n d \pi+\mathcal{O}\left(n^{-1}\right) .
$$

This is (24). To show that $\lambda_{n}=i \tau_{n}^{2}$ is indeed an eigenvalue of $\mathcal{A}$, we have to show that there is a nonzero solution to (5) with $\tau=\tau_{n}$. Let $\phi_{n}$ be the function defined by (16) with $\tau=\tau_{n}$. If $\phi_{n} \neq 0$ then $\lambda_{n}=i \tau_{n}^{2}$ is an eigenvalue of $\mathcal{A}$. If $\phi_{n}=0$, then by (16), it must have

$$
\operatorname{sh} \tau_{n}(1-d) \sin \tau_{n}=s h \tau_{n} \sin \tau_{n}(1-d)=\operatorname{sh} \tau_{n} d \sin \tau_{n}=\operatorname{sh} \tau_{n} \sin \tau_{n} d=0 .
$$


If $\sin \tau_{n} d=0$, then $\tau_{n}$ is a real number and so $s h \tau_{n} d \neq 0$. It follows from above that $\sin \tau_{n}=0$. Therefore, $\phi_{n}(x)=\sin \tau_{n} x$ is a nonzero solution to (5). If $\sin \tau_{n} d \neq 0$, we shall show in (27) below that $\phi_{n} \neq 0$ for $n$ sufficiently large. So for all sufficiently large $n, \lambda_{n}$ are eigenvalues of $\mathcal{A}$.

It should be pointed out that (24) is only an asymptotic expression of "a family of" eigenvalues of $\mathcal{A}$ at the moment, in the next section, however, we shall show that (24) is indeed an asymptotic expression for all eigenvalues of $\mathcal{A}$. This is one of the merits of this approach.

\section{Riesz basis generation}

Let us recall that for a closed linear operator $A$ in a Hilbert space $\mathbf{H}$, a nonzero $x \in \mathbf{H}$ is called a generalized eigenvector of $A$, corresponding to an eigenvalue $\lambda$ of $A$ which has finite algebraic multiplicity, if there is a positive integer $n$ such that $(\lambda-A)^{n} x=0$. A sequence $\left\{x_{n}\right\}_{1}^{\infty}$ in $\mathbf{H}$ is called a Riesz basis for $\mathbf{H}$ if there is an orthonomal basis $\left\{e_{n}\right\}_{1}^{\infty}$ in $\mathbf{H}$ and a linear bounded invertible operator $\mathbf{T}$ such that

$$
\mathbf{T} e_{n}=x_{n}, n=1,2, \ldots .
$$

It is seen that each Riesz basis sequence must be approximately normalized:

$$
C_{1} \leq\left\|x_{n}\right\| \leq C_{2}, C_{1}, C_{2}>0, n=1,2, \ldots .
$$

Suppose that $\left\{\lambda_{n}\right\}_{1}^{\infty} \subset \sigma(A)$. If each $\lambda_{n}$ has finite algebraic multiplicity $m_{n}$, then there is a sequence of linear independent generalized eigenvectors $\left\{x_{n_{i}}\right\}_{i=1}^{m_{n}}$ corresponding to $\lambda_{n}$. If $\left.\left\{\left\{x_{n_{i}}\right\}_{i=1}^{m_{n}}\right\}\right\}_{n=1}^{\infty}$ forms a Riesz basis for $\mathbf{H}$, then $A$ generates a $C_{0}$-semigroup $e^{A t}$ which can be represented as

$$
e^{A t} x=\sum_{n=1}^{\infty} e^{\lambda_{n} t} \sum_{i=1}^{m_{n}} a_{n i} \sum_{j=1}^{m_{n}} f_{n_{i j}}(t) x_{n_{j}}, \text { for any } x=\sum_{n=1}^{\infty} \sum_{i=1}^{m_{n}} a_{n i} x_{n_{i}} \in \mathbf{H}
$$

where $f_{n_{i j}}(t)$ is a polynomial of $t$ with order less than $m_{n}$. In particular, if $a<\operatorname{Re} \lambda<b$ for some reals a and $b$ then $A$ generates a $C_{0}$-group 
on $\mathbf{H}$. Moreover, if $m_{n}$ are uniformly bounded with respect to $n$, then the spectrum-determined growth condition holds for $e^{A t}: \omega(A)=S(A)$, where $\omega(A)$ is the growth order of $e^{A t}$ and $S(A)$ is the spectral bound of $e^{A t}$.

From general operator theory, if $A$ is a self-adjoint or skew-adjoint operator with compact resolvent in $\mathbf{H}$, then there is always a sequence of eigenvectors of $A$ which forms a Riesz (orthogonal, actually) basis for $\mathbf{H}$. Hence for such an operator, the geometric and algebraic multiplicity of each eigenvalue are the same. If $A$ is self-adjoint, then all its eigenvalues are located on the real axis, whereas if $A$ is skew-adjoint, all its eigenvalues lie on the imaginary axis. For a general nonself-adjoint operator, the following result is recently reported in Guo (2001a) and a simpler proof is presented in Guo (2001c).

Theorem 2. Let $A$ be a densely defined discrete operator (that is, $(\lambda-A)^{-1}$ is compact for some $\left.\lambda \in \rho(A)\right)$ in a Hilbert space $\mathbf{H}$. Let $\left\{z_{n}\right\}_{1}^{\infty}$ be a Riesz basis for $\mathbf{H}$. If there are an $N \geq 0$ and a sequence of generalized eigenvectors $\left\{x_{n}\right\}_{N+1}^{\infty}$ of $A$ such that

$$
\sum_{N+1}^{\infty}\left\|x_{n}-z_{n}\right\|^{2}<\infty
$$

then

(i). There are constants $M>N$ and generalized eigenvectors $\left\{x_{n 0}\right\}_{1}^{M}$ of A such that $\left\{x_{n 0}\right\}_{1}^{M} \cup\left\{x_{n}\right\}_{M+1}^{\infty}$ forms a Riesz basis for $\mathbf{H}$.

(ii). Let $\left\{x_{n 0}\right\}_{1}^{M} \cup\left\{x_{n}\right\}_{M+1}^{\infty}$ correspond to eigenvalues $\left\{\sigma_{n}\right\}_{1}^{\infty}$ of $A$. Then $\sigma(A)=\left\{\sigma_{n}\right\}_{1}^{\infty}$, where $\sigma_{n}$ is counted according to its algebraic multiplicity.

(iii). If there is an $M_{0}>0$ such that $\sigma_{n} \neq \sigma_{m}$ for all $m, n>M_{0}$, then there is an $N_{0}>M_{0}$ such that all $\sigma_{n}, n>N_{0}$ are algebraically simple.

Because every sequence of Riesz basis is approximately normalized, in order to apply Theorem 2 to system (1), first, we have to normalize the eigenfunctions. The results are summarized in the following theorem.

Theorem 3. Let $\lambda_{n}=i \tau_{n}^{2}$, where $\tau_{n}$ is determined by (26), be an eigenvalue of $\mathcal{A}$. Then the corresponding eigenfunctions $\left(\phi_{n}, \lambda_{n} \phi_{n}\right)$, where $\phi_{n}$ is defined by (16) with $\tau=\tau_{n}$, have the following asymptotic expression:

$$
2 \tau_{n}^{-2} e^{-\tau_{n}}\left(\sin \tau_{n} d\right)^{-1}\left(\begin{array}{c}
\phi_{n}^{\prime \prime}(x) \\
\lambda_{n} \phi_{n}(x)
\end{array}\right)=(-1)^{n}\left(\begin{array}{c}
1 \\
-i
\end{array}\right) \sin n \pi x+\mathcal{O}\left(n^{-1}\right)
$$


which holds uniformly for $x \in[0,1]$ when $\sin \tau_{n} d \neq 0$. Hence

$$
\lim _{n \rightarrow \infty}\left\|2 \tau_{n}^{-2} e^{-\tau_{n}}\left(\sin \tau_{n} d\right)^{-1}\left(\phi_{n}, \lambda_{n} \phi_{n}\right)\right\|_{\mathcal{H}}^{2}=2 .
$$

In particular, $\phi_{n} \neq 0$ for sufficiently large $n$ when $\sin \tau_{n} d \neq 0$. When $\sin \tau_{n} d=0$, we have shown in the end of the proof of Theorem 1 that $\phi_{n}(x)=\sin \tau_{n} x$ is an eigenfunction of $\mathcal{A}$ corresponding to $\lambda_{n}=i \tau_{n}^{2}$. Therefore, we may consider (27) valid for all sufficiently large $n$.

Proof. We treat only $\phi_{n}(x), 0 \leq x<d$ since other terms can be treated similarly using the following expression of $\phi_{n}^{\prime \prime}$ :

$\tau_{n}^{-2} \phi_{n}^{\prime \prime}(x)=-s h \tau_{n}(1-d) \sin \tau_{n} \operatorname{sh} \tau x-\operatorname{sh} \tau_{n} \sin \tau_{n}(1-d) \sin \tau_{n} x, 0 \leq x<d$.

When $\sin \tau_{n} d \neq 0$,

$$
\begin{aligned}
2 \tau_{n}^{-2} e^{-\tau_{n}}\left(\sin \tau_{n} d\right)^{-1} \phi_{n}^{\prime \prime}(x) & =\frac{\sin \tau_{n}}{\sin d \tau_{n}} \mathcal{O}(1)+\frac{\sin \tau_{n}}{\sin d \tau_{n}} \mathcal{O}(1)+ \\
& +\cos \tau_{n} \sin \tau_{n} x+\mathcal{O}\left(n^{-1}\right)
\end{aligned}
$$

Note that

$$
\left\{\begin{array}{l}
\cos \tau_{n} x=\cos n \pi x+\mathcal{O}\left(n^{-1}\right), \sin \tau_{n} x=\sin n \pi x+\mathcal{O}\left(n^{-1}\right), \\
e^{-\tau_{n} y}=e^{-n \pi y}+\mathcal{O}\left(n^{-1}\right)
\end{array}\right.
$$

which hold uniformly pointwisely for any bounded $y>0$ and $x \in[0,1]$. Moreover, it follows from (17) that

$$
\frac{\sin \tau_{n}}{\sin d \tau_{n}}=\frac{-\mathcal{K} i s h \tau_{n} \sin \tau_{n}(1-d)}{2 \tau_{n} \operatorname{sh} \tau_{n}-K i s h \tau_{n}(1-d) \operatorname{sh} \tau_{n} d}=\mathcal{O}\left(n^{-1}\right)
$$

By (28) and (29), we have

$$
\begin{aligned}
2 \tau_{n}^{-2} e^{-\tau_{n}}\left(\sin \tau_{n} d\right)^{-1} \phi_{n}^{\prime \prime}(x) & =\cos \tau_{n} \sin \tau_{n} x+\mathcal{O}\left(n^{-1}\right) \\
& =\cos n \pi \sin n \pi x+\mathcal{O}\left(n^{-1}\right) \\
& =(-1)^{n} \sin n \pi x+\mathcal{O}\left(n^{-1}\right) .
\end{aligned}
$$

This is (27). 
Now we are in a position to develop the basis property for system (1) by applying Theorem 2 . Let

$$
\Phi_{n}=2(-1)^{n+1} \tau_{n}^{-2} e^{-\tau_{n}}\left(\sin d \tau_{n}\right)^{-1}\left(\phi_{n}, \lambda_{n} \phi_{n}\right)
$$

be the eigenfunctions of $\mathcal{A}$ corresponding to $\lambda_{n}=i \tau_{n}^{2}$ claimed in Theorem 3. Let

$$
\Psi_{n}=\left(\sin n \pi x /(n \pi)^{2}, i \sin n \pi x\right), n \geq 1 .
$$

All $\Psi_{n}$ and their conjugates are composed of all eigenfunctions of $\mathcal{A}_{0}$, which form a Riesz basis of $\mathcal{H}$. It follows from Theorem 3 that there is an $N>0$ such that

$$
\sum_{n>N}^{\infty}\left\|\Psi_{n}-\Phi_{n}\right\|_{\mathcal{H}}^{2}=\sum_{n>N}^{\infty} \mathcal{O}\left(n^{-2}\right)<\infty .
$$

The same thing is true for the conjugates. Therefore, Theorem 2 is applicable and we obtain our main result of this paper.

Theorem 4. For any real number $K$

(i) there is a sequence of generalized eigenfunctions of operator $\mathcal{A}$, which forms a Riesz basis for the state space $\mathcal{H}$;

(ii) (24) is an asymptotic expression for all eigenvalues of $\mathcal{A}$;

(iii) all eigenvalues of $\mathcal{A}$ with sufficiently large modulus are algebraically simple, therefore, $\mathcal{A}$ generates a $C_{0}$-group on $\mathcal{H}$ and the spectrumdetermined growth condition holds for the $C_{0}$-semigroup $e^{\mathcal{A} t}$ generated by $\mathcal{A}: \omega(\mathcal{A})=S(\mathcal{A})$.

Remark 1. To conclude that (24) is indeed an asymptotic expression for all eigenvalues of $\mathcal{A}$, we do not need necessarily Theorem 2. Actually, it can follow from (27) and (30) and Bari's theorem that a sequence in a Hilbert space is a Riesz basis if it is quadratically close to a given Riesz basis, because a nontrivial subset of a basis can not be a basis and any finitely linearly independent generalized eigenvectors of a discrete operator in a Hilbert space must be $\omega$-linearly independent (see, e.g., Singer, 1975).

\section{$5 \quad$ Exponential stability}

This section is devoted to the stability analysis of system (1). Because the spectrum-determined condition is valid for system (1), the stability 
of the system is determined by the distribution of eigenvalues. First, by Theorem 4 and (24), a necessary condition for system (1) to be asymptotically stable is $K>0$.

Proposition 1. Assume that $K>0$. Then system (1) is asymptotically stable if and only if $d$ is an irrational number.

Proof. A simple calculation shows that

$$
R e<\mathcal{A}(f, g),(f, g)>=-K|g(d)|^{2} .
$$

Hence $\mathcal{A}$ is dissipative for any $K>0$. Because $\mathcal{A}$ is a discrete operator, $e^{\mathcal{A} t}$ is asymptotically stable if and only if $\operatorname{Re} \lambda \neq 0$ for any $\lambda \in \sigma(\mathcal{A})$. Let $(\phi(x), \lambda \phi(x))$ be an eigenfunction corresponding to $\lambda$, where $\phi$ satisfies (5). Then a simple calculation shows that $\operatorname{Re} \lambda=0$ if and only if $\phi(d)=0$ for a nonzero $\phi$ satisfying

$$
\left\{\begin{array}{l}
\phi^{(4)}(x)-\omega^{4} \phi(x)=0,0<x<1 \\
\phi(0)=\phi^{\prime \prime}(0)=\phi(1)=\phi^{\prime \prime}(1)=0
\end{array}\right.
$$

for some $\omega>0$ satisfying $\sin \omega=0$. that is, $d$ is a nodal point of the free system (22). The solution of (30) is $\phi(x)=\sin \omega x$ (up to a scalar). Combining $\sin \omega=0$ and $\phi(d)=\sin \omega d=0$ shows that $d$ is a rational number.

Suppose that $d$ is an irrational number. We want to know when the system is exponentially stable. It follows from Theorem 4 that system (1) is asymptotically stable but not exponentially stable if and only if

$$
\sin n d \pi \rightarrow 0
$$

for some positive integer sequence $\{n\}$ approaching infinity. In order to derive condition (31) with respect to $d$, we need a result on the approximation of irrational by rationals.

Lemma 3. [9, Theorem 438]: If $d$ is irrational, $\alpha$ is arbitrary and $N$ and $\epsilon$ are positive, then there are integers $n$ and $p$ such that $n>N$ and

$$
|n d-p-\alpha|<\epsilon
$$


Theorem 5. Suppose that $\mathcal{K}>0$. Then system (1) is not exponentially stable for any $0<d<1$.

Proof. We need only show that (31) holds for any irrational $d$.

For irrational $d$, it follows from Lemma 3 that for any $0<\epsilon<\pi / 2$ and $N>0$, there are integers $n, p, n>N$ such that

$$
|n d-p|<\epsilon \text {. }
$$

Hence

$$
|\sin d n \pi|=|\sin (n d-p+p) \pi|=|\sin (n d-p) \pi \cos p \pi| \leq \epsilon .
$$

Therefore, there exists a sequence $\{n\}$ approaching infinity such that

$$
\sin n d \pi \rightarrow 0 \text {. }
$$

The proof is complete.

The proof of Theorem 5 which relies mainly on the asymptotic expression (24) of eigenvalues gives us much more information on the distribution of eigenvalues than exponential stability itself. Let us investigate a special case where $d=1 / 2$. In this case, it follows from (24) that

$$
R e \lambda \rightarrow-K \sin ^{2} n \pi / 2
$$

as $|\lambda| \rightarrow \infty$. There are two branches of eigenvalues. One branch approaches imaginary axis and another approaches $\operatorname{Re} \lambda=-K$. This property was also observed in Chen et al. (1989) for the same equation with different joint feedback and boundary conditions. However, we shall explain by Example 1 in the next section that this phenomenon is not intrinsic for beam equation with middle joint feedback control.

Moreover, if $d$ is not the middle point, the situation becomes much more complicated. If $d=\frac{p}{q}$ is a rational number for some coprime integers $p, q$, then for any integer $n$, there are integers $w, k$ such that

$$
n=w q+k, 0 \leq k<q .
$$

Hence

$$
\sin ^{2} n d \pi=\sin ^{2} k d \pi
$$


where $0 \leq k<q$, and so the eigenvalues may have at most $q$ branches as follows:

$$
R e \lambda \rightarrow-K \sin ^{2} k d \pi, \quad k=0,1,2, \cdots, q-1 .
$$

If $d$ is irrational, similar to (32), we have, for any given $\theta>0$

$$
\begin{aligned}
& \sin ^{2} n d \pi=\sin ^{2}(n d-p-\theta+p+\theta) \pi \\
& =[\sin (n d-p-\theta) \pi \cos (p+\theta) \pi+\cos (n d-p-\theta) \pi \sin (p+\theta) \pi]^{2}
\end{aligned}
$$

for any integer $p$. By Lemma 3, we can choose sufficiently large $n$ and $p$ such that $n d-p-\theta$ is as small as possible. Therefore, for any $\theta$, there exists a sequence of positive integers $\{n\}$ approaching infinity such that

$$
R e \lambda_{n} \rightarrow-K \sin ^{2} \theta \pi \text {. }
$$

That is, the asymptotes of eigenvalues are dense in

$$
\{z \mid-K \leq \operatorname{Re} z \leq 0\} .
$$

This is not unexpected because, for a string vibration equation with joint feedback control, when the system is asymptotically but not exponentially stable which is usually true for an irrational joint point $d$ (Zhu et al. ,1997), then there are infinite number of asymptotes of eigenvalues. This is a consequence of a general property for zeros of analytic almost periodic functions (see, e.g., Lemma 3.2 in Henry, 1975).

\section{Additional examples}

To demonstrate the approach further, we investigate two more examples. Both of them have been studied in Rebarber (1995) and Chen et al. (1989) respectively, for which exponential stability and the asymptotic distribution of eigenvalues are already known from the articles cited. Here we show that more profound results such as the Riesz basis generation and spectrum-determined growth condition are also true for these systems, we omit the computation process because the proof can be done easily by following the procedures for system (1). We particularly pay more attention to the distribution of eigenvalues. It will be seen that our treatment greatly improves and simplifies the proof by other existing approaches. 
For notational simplicity, we use, in the sequel, $\lambda=i \tau^{2}$ or $\lambda_{n}=i \tau_{n}^{2}$ to denote the eigenvalues of the controlled systems and by $\mu=i \omega^{2}$ or $\mu_{n}=i \omega_{n}^{2}$ to denote those of the associated auxiliary free Riesz systems.

Example 1. The first equation in Rebarber (1995) is just equation (1) but with different boundary conditions:

$$
\left\{\begin{array}{l}
y_{t t}(x, t)+y_{x x x x}(x, t)=0,0<x<d, d<x<1, \\
y(0, t)=y_{x x}(0, t)=0, y_{x}(1, t)=y_{x x x}(1, t)=0, \\
y\left(d^{+}, t\right)=y\left(d^{-}, t\right), y_{x}\left(d^{+}, t\right)=y_{x}\left(d^{-}, t\right), y_{x x}\left(d^{+}, t\right)=y_{x x}\left(d^{-}, t\right), \\
y_{x x x}\left(d^{-}, t\right)-y_{x x x}\left(d^{+}, t\right)=K y_{t}(d, t) .
\end{array}\right.
$$

Similar to (2) we can write (38) as an evolution equation in the state Hilbert space $H=H_{e}^{2}(0,1) \times L^{2}(0,1)$ :

$$
\frac{d}{d t} Y(t)=\mathcal{B} Y(t)
$$

where $Y(t)=\left(y(\bullet, t), y_{t}(\bullet, t)\right) \in H$ and $\mathcal{B}$ is defined by

$$
\left\{\begin{aligned}
\mathcal{B}(f, g)= & \left(g(x),-f^{(4)}(x)\right), \forall(f, g) \in D(\mathcal{B}), \\
D(\mathcal{B})=\left\{(f, g) \in H_{e}^{2}(0,1) \times H_{e}^{2}(0,1)|f|_{[0, d]} \in H^{4}(0, d),\right. & \\
& \left.f\right|_{[d, 1]} \in H^{4}(d, 1), \\
& f^{\prime \prime}\left(d^{-}\right)=f^{\prime \prime}\left(d^{+}\right), f^{\prime \prime \prime}\left(d^{-}\right)-f^{\prime \prime \prime}\left(d^{+}\right)=K g(d), \\
& \left.f^{\prime \prime}(0)=f^{\prime \prime \prime}(1)=0\right\}
\end{aligned}\right.
$$

where $H_{e}^{2}(0,1)=\left\{f \in H^{2}(0,1) \mid f(0)=f^{\prime}(1)=0\right\}$ with the inner product induced norm:

$$
\|f\|_{H_{e}^{2}}^{2}=\int_{0}^{1}\left|f^{\prime \prime}(x)\right| d x .
$$

With almost the same computation process as we have done for system (1), we can get the parallel results for system (38).

Proposition 2. (i). $\mathcal{B}^{-1}$ exists and is compact.

(ii). For each $\lambda=i \tau^{2} \in \sigma(\mathcal{B})$, an eigenfunction corresponding to $\lambda$ is $(\phi, \lambda \phi)$ if $\phi \neq 0$, where

$$
\phi(x)=\left\{\begin{array}{l}
-\operatorname{ch} \tau(1-d) \cos \tau \operatorname{sh} \tau x+\operatorname{ch} \tau \cos \tau(1-d) \sin \tau x, 0 \leq x<d, \\
-\operatorname{sh} \tau d \cos \tau \operatorname{ch} \tau(1-x)+\operatorname{ch} \tau \sin \tau d \cos \tau(1-x), d<x \leq 1,
\end{array}\right.
$$


satisfying

$$
\left\{\begin{array}{l}
\lambda^{2} \phi(x)+\phi^{(4)}(x)=0,0<x<d, d<x<1, \\
\phi(0)=\phi^{\prime \prime}(0)=\phi^{\prime}(1)=\phi^{\prime \prime \prime}(1)=0, \\
\phi\left(d^{-}\right)=\phi\left(d^{+}\right), \phi^{\prime}\left(d^{-}\right)=\phi^{\prime}\left(d^{+}\right), \phi^{\prime \prime}\left(d^{-}\right)=\phi^{\prime \prime}\left(d^{+}\right), \\
\phi^{\prime \prime \prime}\left(d^{-}\right)-\phi^{\prime \prime \prime}\left(d^{+}\right)=K \lambda \phi(d) .
\end{array}\right.
$$

Each eigenfunction associated with $\lambda$ takes the form $(\phi(x), \lambda \phi(x))$ where $\phi$ is a nonzero solution to (42). The characteristic equation that $\lambda$ satisfies is

$$
2 \tau c h \tau \cos \tau=\mathcal{K} i[\operatorname{ch} \tau(1-d) \operatorname{sh} \tau d \cos \tau-\operatorname{ch} \tau \cos \tau(1-d) \sin \tau d] .
$$

(iii). There is a family of eigenvalues of $\mathcal{B}$ having the following asymptotic expression:

$$
\lambda_{n}=i \tau_{n}^{2}=i(m \pi)^{2}-K \sin ^{2} m \pi d+\mathcal{O}\left(n^{-1}\right)
$$

for all sufficiently large integers $n$, where $m=n-1 / 2$.

It follows from (43) and (44) that when $\sin \tau_{n} d \neq 0$,

$$
\frac{\cos \tau_{n}}{\sin \tau_{n} d}=\mathcal{O}\left(\left|\tau_{n}\right|^{-1}\right) .
$$

Let $\phi_{n}$ be the function defined by (41) with $\tau=\tau_{n}$. Then by similar arguments as (27), we can obtain

$$
2(-1)^{n} \tau_{n}^{-2}\left(\sin \tau_{n} d\right)^{-1} e^{-\tau_{n}}\left(\begin{array}{c}
\phi_{n}^{\prime \prime}(x) \\
\lambda_{n} \phi_{n}(x)
\end{array}\right)=\sin m \pi x\left(\begin{array}{c}
-1 \\
i
\end{array}\right)+\mathcal{O}\left(n^{-1}\right)
$$

which holds uniformly for $x \in[0,1]$. When $\phi_{n}=0$, it follows from (41) that

$\operatorname{ch} \tau_{n}(1-d) \cos \tau_{n}=\operatorname{ch} \tau_{n} \cos \tau_{n}(1-d)=\operatorname{sh} \tau_{n} d \cos \tau_{n}=\operatorname{ch} \tau_{n} \sin \tau_{n} d=0$.

It is seen that if $\sin \tau_{n} d=0$, then $\tau_{n}$ is a real number and so $s h \tau_{n} \neq 0$. Hence $\cos \tau_{n}=0$. A direct computation shows that $\phi_{n}=\sin \tau_{n} x$ is a nonzero solution to (42) and hence $\left(\sin \tau_{n} x, i \tau_{n}^{2} \sin \tau_{n} x\right)$ is an eigenfunction corresponding to $\lambda_{n}=i \tau_{n}^{2}$. So same as (27), we may consider (45) to be valid for all sufficiently large $n$. 
Let

$$
\Phi_{n}(x)=2(-1)^{n} \tau_{n}^{-2}\left(\sin \tau_{n} d\right)^{-1} e^{-\tau_{n}}\left(\phi_{n}, \lambda_{n} \phi_{n}(x)\right) .
$$

Then it follows from (45) that there is an $N>0$ such that

$$
\sum_{n>N}^{\infty}\left\|\Phi_{n}(x)-\Psi_{n}(x)\right\|^{2}<\infty
$$

where $\Psi_{n}(x)=\left\{\sin m \pi x /(m \pi)^{2}, i \sin m \pi x\right\}_{1}^{\infty}$. The same thing is true for the conjugates. Because $\left\{\Psi_{n}(x), \bar{\Psi}_{n}(x)\right\}_{1}^{\infty}$ forms a Riesz basis in $\mathbf{H}$, Theorem 2 is applicable and we obtain the following paralleling results for system (38) with Theorem 4.

Theorem 6. For any real number $K$

(i) there is a sequence of generalized eigenfunctions of operator $\mathcal{B}$, which forms a Riesz basis for the state space $H$;

(ii) (44) is an asymptotic expression for all eigenvalues of $\mathcal{B}$;

(iii) all eigenvalues of $\mathcal{B}$ with sufficiently large modulus are algebraically simple; therefore, $\mathcal{B}$ generates a $C_{0}$-group on $H$ and the spectrumdetermined growth condition holds for the $C_{0}$-semigroup $e^{\mathcal{B} t}$ generated by $\mathcal{B}: \omega(\mathcal{B})=S(\mathcal{B})$.

The following conclusions are obtained in Theorem 1.1 of Rebarber (1995). Here we give a simple proof based on Theorem 6 .

Corollary 1. Suppose $K>0$. Then

(i). System (38) is asymptotically stable if and only if $d$ is either a irrational number or a rational number so that

$$
d \neq \frac{2 s}{2 k-1}
$$

for any integers $s, k$.

(ii). System (38) is exponentially stable if and only if it is asymptotically stable and $d$ is not an irrational number.

Proof. Similar to operator $\mathcal{A}$, when $K>0, \mathcal{B}$ is dissipative. $e^{\mathcal{B} t}$ is asymptotically stable if and only if $\operatorname{Re} \lambda \neq 0$ for any $\lambda \in \sigma(\mathcal{B})$. Let $(\phi(x), \lambda \phi(x))$ be the eigenfunction corresponding to $\lambda=i \tau^{2}$ which is a nonzero solution of (42). Then a simple calculation shows that $\operatorname{Re} \lambda=0$ 
if and only if $\phi(d)=0$ for some positive $\tau$ satisfying $\cos \tau=0$. Meanwhile, a simple calculation shows that in this case $\phi(x)=\sin \tau x$. Combining $\cos \tau=0$ and $\sin \tau d=0$ gives

$$
d=\frac{2 s}{2 k-1}
$$

for some positive integers $s, k$, proving (i). Next, by (ii) of Theorem 6 , we know that when $K>0$ system (38) is asymptotically stable but not exponentially stable if and only if

$$
\sin ^{2}(n-1 / 2) d \pi \rightarrow 0
$$

for some positive integers sequence $\{n\}$ approaching infinity. We distinguish two cases as in Theorem 5 :

(i). $d=p / q$ is a rational number for some coprime positive integers $p$ and $q$. In this case, writing any positive integer $n=w q+k$ for some integers $w$ and $k, 0 \leq k<q$, we have $\sin ^{2}\left(n-\frac{1}{2}\right) d \pi=\sin ^{2}\left(k-\frac{1}{2}\right) d \pi$. Hence condition (46) holds if and only if $\left(k-\frac{1}{2}\right) d \pi=s \pi$ for some integer $s$, that is $d=\frac{2 s}{2 k-1}$. Hence the system is not asymptotically stable.

(ii). If $d$ is irrational, a similar treatment as in Theorem 5 (see also Lemma 2.9 in Rebarber, 1995) shows that (46) is always valid for some positive integers sequence. Therefore, system (38) is never exponentially stable for any irrational joint $d$.

Finally, we indicate an interesting fact for system (38). When $d=$ $1 / 2$, that is the stabilizer is installed at the middle of the beam as it was studied in Chen et al. $(1989,1990)$, we see from (ii) of Theorem 6 that

$$
R e \lambda_{n} \rightarrow-K \sin ^{2} \pi / 4=-\frac{K}{2}, \text { as } n \rightarrow \infty .
$$

Hence there is only one family of eigenvalues. This is different from the cases studied in Chen et al. $(1989,1990)$ that the middle joint stabilizer for Euler-Bernoulli beams produces most often two families of eigenvalues. 
Example 2. Our next example is a special case of the equation considered in Chen et al. $(1989,1990)$ :

$$
\left\{\begin{array}{l}
y_{t t}(x, t)+y_{x x x x}(x, t)=0,0<x<d, d<x<1, \\
y(0, t)=y_{x}(0, t)=0, y_{x x}(1, t)=y_{x x x}(1, t)=0, \\
y_{x}\left(d^{+}, t\right)=y_{x}\left(d^{-}, t\right), y_{x x}\left(d^{+}, t\right)=y_{x x}\left(d^{-}, t\right) \\
y_{x x x}\left(d^{-}, t\right)=y_{x x x}\left(d^{+}, t\right) \\
y_{t}\left(d^{-}, t\right)-y_{t}\left(d^{+}, t\right)=K y_{x x x}(d, t)
\end{array}\right.
$$

where $d=1 / 2$. Define the energy state space $\mathbf{H}$ for system (47): $\mathbf{H}=H_{p}^{2}(0,1) \times L^{2}(0,1)$, where $H_{p}^{2}(0,1)=\left\{\left.f\right|_{[0, d]} \in H^{2}(0, d),\left.f\right|_{[d, 1]} \in\right.$ $\left.H^{2}(d, 1) \mid f(0)=f^{\prime}(0)=0, f^{\prime}\left(d^{-}\right)=f^{\prime}\left(d^{+}\right)\right\}$. Then system (47) can be written as an evolutionary equation in $\mathbf{H}$ :

$$
\frac{d}{d t} Y(t)=\mathcal{C} Y(t)
$$

where $Y(t)=\left(y(\bullet, t), y_{t}(\bullet, t)\right) \in \mathbf{H}$ and $\mathcal{C}$ is defined by

$$
\left\{\begin{aligned}
\mathcal{C}(f, g)= & \left(g(x),-f^{(4)}(x)\right), \forall(f, g) \in D(\mathcal{C}) \\
D(\mathcal{C})=\left\{(f, g) \mid\left\{\left.(f, g)\right|_{[0, d]} \in H^{4}(0, d) \cap H^{2}(0, d)\right.\right. & \\
& \left.(f, g)\right|_{[d, 1]} \in H^{4}(d, 1) \cap H^{2}(d, 1) \\
& f(0)=f^{\prime}(0)=f^{\prime \prime}(1)=f^{\prime \prime \prime}(1)=g(0)=g^{\prime}(0)=0 \\
& f^{\prime}\left(d^{-}\right)=f^{\prime}\left(d^{+}\right), g^{\prime}\left(d^{-}\right)=g^{\prime}\left(d^{+}\right) \\
& f^{\prime \prime}\left(d^{-}\right)=f^{\prime \prime}\left(d^{+}\right), f^{\prime \prime \prime}\left(d^{-}\right)=f^{\prime \prime \prime}\left(d^{+}\right) \\
& \left.\left.g^{(} d^{-}\right)-g\left(d^{+}\right)=K f^{\prime \prime \prime}(d)\right\}
\end{aligned}\right.
$$

In this example, the function $\phi$ corresponding to (16) takes the following form:

$$
\phi(x)=F(x)+\left\{\begin{array}{l}
-\operatorname{ch} \tau(x-d)-\cos \tau(x-d)-\operatorname{ch} \tau \cos \tau(1-d+x) \\
-\operatorname{ch} \tau(1-d+x) \cos \tau, 0 \leq x<d \\
\operatorname{ch} \tau(x-d)+\cos \tau(x-d)+\operatorname{ch} \tau \cos \tau(1+d-x) \\
+\operatorname{ch} \tau(1+d-x) \cos \tau, d<x \leq 1
\end{array}\right.
$$

where

$$
\begin{aligned}
F(x) & =\operatorname{ch} \tau d \cos \tau x-\operatorname{sh} \tau d \sin \tau x+\operatorname{ch} \tau x \cos \tau d+\operatorname{sh} \tau x \sin \tau d \\
& -\operatorname{sh} \tau \sin \tau(1-d-x)+\operatorname{sh} \tau(1-d-x) \sin \tau \\
& +\operatorname{sh} \tau(1-x) \sin \tau(1-d)+\operatorname{ch} \tau(1-x) \cos \tau(1-d) \\
& +\operatorname{ch} \tau(1-d) \cos \tau(1-x)-\operatorname{sh} \tau(1-d) \sin \tau(1-x), 0 \leq x \leq 1 .
\end{aligned}
$$


And the characteristic equation is

$$
\begin{aligned}
4+4 \operatorname{ch} \tau \cos \tau & =K i \tau[-\operatorname{ch} \tau \sin \tau-\operatorname{sh} \tau \cos \tau+2 \operatorname{ch} \tau d \sin \tau d \\
& +2 \operatorname{sh} \tau d \cos \tau d \\
& -\operatorname{sh} \tau \cos \tau(1-2 d)-\operatorname{ch} \tau(1-2 d) \sin \tau \\
& -2 \operatorname{ch} \tau(1-d) \sin \tau(1-d) \\
& -2 \operatorname{sh} \tau(1-d) \cos \tau(1-d)] .
\end{aligned}
$$

From (52), the left hand side is a lower perturbation of the right hand side, so we should choose the following auxiliary free system by letting $1 / K=0$ in $(47)$ :

$$
\left\{\begin{array}{l}
y_{t t}(x, t)+y_{x x x x}(x, t)=0,0<x<d, d<x<1, \\
y(0, t)=y_{x}(0, t)=0, y_{x x}(1, t)=y_{x x x}(1, t)=0, \\
y_{x}\left(d^{+}, t\right)=y_{x}\left(d^{-}, t\right), y_{x x}\left(d^{+}, t\right)=y_{x x}\left(d^{-}, t\right), \\
y_{x x x}\left(d^{-}, t\right)=y_{x x x}\left(d^{+}, t\right)=0 .
\end{array}\right.
$$

The system operator $\mathcal{C}_{0}$ of $(55)$ is operator $\mathcal{C}$ with $1 / K=0$, that is

$$
\left\{\begin{aligned}
\mathcal{C}_{0}(f, g)= & \left(g(x),-f^{(4)}(x)\right), \forall(f, g) \in D\left(\mathcal{C}_{0}\right), \\
D\left(\mathcal{C}_{0}\right)= & =\left\{(f, g) \mid\left\{\left.(f, g)\right|_{[0, d]} \in H^{4}(0, d) \cap H^{2}(0, d),\right.\right. \\
& \left.(f, g)\right|_{[d, 1]} \in H^{4}(d, 1) \cap H^{2}(d, 1), \\
& f(0)=f^{\prime}(0)=f^{\prime \prime}(1)=f^{\prime \prime \prime}(1)=g(0)=g^{\prime}(0)=0, \\
& f^{\prime}\left(d^{-}\right)=f^{\prime}\left(d^{+}\right), g^{\prime}\left(d^{-}\right)=g^{\prime}\left(d^{+}\right), \\
& \left.f^{\prime \prime}\left(d^{-}\right)=f^{\prime \prime}\left(d^{+}\right), f^{\prime \prime \prime}\left(d^{-}\right)=f^{\prime \prime \prime}\left(d^{+}\right)=0\right\} .
\end{aligned}\right.
$$

The characteristic equation of (53) can be obtained by letting the right hand side of (52) be zero:

$$
\begin{aligned}
& -\operatorname{ch} \omega \sin \omega-\operatorname{sh} \omega \cos \omega+2 \operatorname{ch} \omega d \sin \omega d+2 \operatorname{sh} \omega d \cos \omega d \\
& -\operatorname{sh} \omega \cos \omega(1-2 d)-\operatorname{ch} \omega(1-2 d) \sin \omega \\
& -2 \operatorname{ch} \omega(1-d) \sin \omega(1-d)-2 \operatorname{sh} \omega(1-d) \cos \omega(1-d)=0 .
\end{aligned}
$$

Finding eigenvalues of operator $\mathcal{C}_{0}$ is equivalent to finding nonnegative solutions of (55). Let

$$
\sigma\left(\mathcal{C}_{0}\right)=\left\{i \omega^{2},-i \omega^{2}\right\}
$$

where $\omega$ is a positive solution of (55). Writing (55) asymptotically for large positive $\omega$, we have

$$
\sin \omega+\cos \omega+\cos \omega(1-2 d)=\mathcal{O}\left(e^{-\gamma \omega}\right), \text { for some } \gamma>0 .
$$


Since $d=1 / 2,(57)$ is just

$$
\sin \omega+\cos \omega=-1+\mathcal{O}\left(e^{-\gamma \omega}\right), \text { for some } \gamma>0 .
$$

There are two branches of solutions to (58):

$$
\left\{\begin{array}{l}
\text { The first branch: } \omega_{n}=2 n \pi-\pi+\mathcal{O}\left(n^{-1}\right) \\
\text { The second branch: } \omega_{n}=2 n \pi-\frac{1}{2} \pi+\mathcal{O}\left(n^{-1}\right)
\end{array}\right.
$$

where $n$ is a large positive integer. Particular attention should be paid to (59) which is an asymptotic expression for all eigenvalues of $\mathcal{C}_{0}$.

Same as before, in a small neighborhood of $\omega_{n}$, we write (52) to be

$$
\sin \tau+\cos \tau+1=\frac{4 i}{K \tau} \cos \tau+\mathcal{O}\left(e^{-\gamma R e \tau}\right), \text { for some } \gamma>0 .
$$

By Rouche's theorem again, we first find a solution of (60) as

$$
\tau_{n}=\omega_{n}+\mathcal{O}\left(n^{-1}\right) .
$$

And then substituting (61) into (60) yields

$$
\left(\cos \omega_{n}-\sin \omega_{n}\right) \mathcal{O}\left(n^{-1}\right)=\frac{4 i}{K \omega_{n}} \cos \omega_{n}+\mathcal{O}\left(n^{-2}\right) .
$$

With $\omega_{n}=2 n \pi-\pi$, we have $\mathcal{O}\left(n^{-1}\right)=\frac{4 i}{K \omega_{n}}+\mathcal{O}\left(n^{-2}\right)$; hence $\tau_{n}=$ $\omega_{n}+\frac{4 i}{\mathcal{K} \omega_{n}}+\mathcal{O}\left(n^{-2}\right)$, and $\lambda_{n}=-\frac{8}{K}+i \omega_{n}^{2}+\mathcal{O}\left(n^{-1}\right)$. With $\omega_{n}=2 n \pi-$ $\frac{1}{2} \pi$, we have $\mathcal{O}\left(n^{-1}\right)=\mathcal{O}\left(n^{-2}\right)$, and $\lambda_{n}=i \omega_{n}^{2}+\mathcal{O}\left(n^{-2}\right)$. Therefore, the two branches of eigenvalues of system (47) have the following asymptotic expression:

$$
\lambda_{n}=\left\{\begin{array}{l}
-8 / K+i[(2 n-1) \pi]^{2}+\mathcal{O}\left(n^{-1}\right) \\
i[(2 n-1 / 2) \pi]^{2}+\mathcal{O}\left(n^{-2}\right)
\end{array}\right.
$$

The estimates (63) are exactly consistent with the main theorem of section 2 in Chen et al. (1989) with $c_{1}=c_{2}=k_{2}^{2}=0, E I=m=1$. However, to explain (63) reasonably, we have to estimate the corresponding eigenfunctions. This is summarized in the following theorem.

Theorem 7. Suppose that $d=1 / 2$. Then for any real number $K$

(i) there is a sequence of generalized eigenfunctions of operator $\mathcal{C}$, which forms a Riesz basis for the state space $\mathbf{H}$; 
(ii) (63) is an asymptotic expression for all eigenvalues of $\mathcal{C}$;

(iii) all eigenvalues of $\mathcal{C}$ with sufficiently large modulus are algebraically simple; therefore, $\mathcal{C}$ generates a $C_{0}$-group on $\mathbf{H}$ and the spectrumdetermined growth condition holds for the $C_{0}$-semigroup $e^{\mathcal{C} t}$ generated by $\mathcal{C}: \omega(\mathcal{C})=S(\mathcal{C})$.

Proof. Let $\lambda_{n}=i \tau_{n}^{2}$ be defined as in (63) where $\tau_{n}$ satisfies (61). Let $\phi_{n}$ be the function defined by (50) and (51) with $\tau=\tau_{n}$. By virtue of (28), we have

$$
\begin{aligned}
& 2 \tau_{n}^{-2} e^{-\tau_{n}} \phi_{n}^{\prime \prime}(x)=e^{-\tau_{n}(1-x)}\left(\cos \tau_{n} d+\sin \tau_{n} d\right) \\
& +\sin \tau_{n}(1-d-x)+\cos \tau_{n}(1-d+x) \\
& +e^{-\tau_{n} x}\left[\sin \tau_{n}(1-d)+\cos \tau_{n}(1-d)\right] \\
& -e^{-\tau_{n}(d-x)} \cos \tau_{n}+\mathcal{O}\left(n^{-1}\right) \\
& =e^{-\tau_{n}(1-x)}\left(\cos \tau_{n} d+\sin \tau_{n} d\right) \\
& +\left(\sin \tau_{n} d+\cos \tau_{n} d\right)\left(\cos \tau_{n} x-\sin \tau_{n} x\right) \\
& +e^{-\tau_{n} x}\left[\sin \tau_{n} d+\cos \tau_{n} d\right]-e^{-\tau_{n}(d-x)} \cos \tau_{n}+\mathcal{O}\left(n^{-1}\right) \\
& =\sin \omega_{n} x-\cos \omega_{n} x-e^{-\omega_{n}(1-x)}-e^{-\omega_{n} x} \\
& -e^{-\omega_{n}(d-x)} \cos \omega_{n}+\mathcal{O}\left(n^{-1}\right) .
\end{aligned}
$$

Similarly

$$
\begin{aligned}
& 2 \tau_{n}^{-2} e^{-\tau_{n}} \phi_{n}(x)=-\cos \tau_{n}(1-d+x)-\sin \tau_{n}(1-d-x) \\
& +e^{-\tau_{n}(1-x)}\left(\cos \tau_{n} d+\sin \tau_{n} d\right) \\
& +e^{-\tau_{n} x}\left[\sin \tau_{n}(1-d)+\cos \tau_{n}(1-d)\right] \\
& -e^{-\tau_{n}(d-x)} \cos \tau_{n}+\mathcal{O}\left(n^{-1}\right) \\
& =-\left(\sin \omega_{n} x-\cos \omega_{n} x\right)-e^{-\omega_{n}(1-x)}-e^{-\omega_{n} x} \\
& -e^{-\omega_{n}(d-x)} \cos \omega_{n}+\mathcal{O}\left(n^{-1}\right) .
\end{aligned}
$$

In particular, $\phi_{n} \neq 0$ for all sufficiently large $n$. Hence (63) is indeed a family of eigenvalues of $\mathcal{C}$. We note that $(a)$ (59) represents all but a finite set of eigenvalues of $\mathcal{C}$; $(b)(64)$ and (65) are also valid for eigenfunctions of $\mathcal{C}_{0}$, that is if $\left(\psi_{n}, i \omega_{n}^{2} \psi_{n}\right)$ is an eigenfunction of $\mathcal{C}_{0}$ corresponding to eigenvalues $i \omega_{n}^{2}$ where $\psi_{n}$ is defined by (50) and (51) with $\tau=\omega_{n}$ then 


$$
\begin{aligned}
& 2 \omega_{n}^{-2} e^{-\omega_{n}}\left(\begin{array}{c}
\psi_{n}^{\prime \prime}(x) \\
i \omega_{n}^{2} \psi(x)
\end{array}\right)= \\
& \left(\begin{array}{c}
\sin \omega_{n} x-\cos \omega_{n} x-e^{-\omega_{n}(1-x)}-e^{-\omega_{n} x}-e^{-\omega_{n}(d-x)} \cos \omega_{n} \\
i\left[-\sin \omega_{n} x+\cos \omega_{n} x-e^{-\omega_{n}(1-x)}-e^{-\omega_{n} x}-e^{-\omega_{n}(d-x)} \cos \omega_{n}\right]
\end{array}\right) \\
& +\mathcal{O}\left(n^{-1}\right),
\end{aligned}
$$

and

$$
\lim _{n \rightarrow \infty}\left\|2 \omega_{n}^{-2} e^{-\omega_{n}}\left(\psi_{n}, i \omega_{n}^{2} \psi_{n}\right)\right\|^{2}=2 ;
$$

(c) all $\left(\psi_{n}, i \omega_{n}^{2} \psi_{n}\right)$ but a finte set are composed of all eigenfunctions of $\mathcal{C}_{0} ;(d) \mathcal{C}_{0}$ is a discrete operator with compact resolvent in $\mathbf{H}$. We may assume without loss of generality that

$$
2 \omega_{n}^{-2} e^{-\omega_{n}}\left(\psi_{n}, i \omega_{n}^{2} \psi_{n}\right), n=1,2, \cdots
$$

with their conjugates are all eigenfunctions of $\mathcal{C}_{0}$ which form a Riesz basis for $\mathbf{H}$. By (64), (65) and (66), there is an $N>0$ such that

$$
\sum_{n>N}^{\infty}\left\|2 \tau_{n}^{-2} e^{-\tau_{n}}\left(\phi_{n}, i \tau_{n}^{2} \phi_{n}\right)-2 \omega_{n}^{-2} e^{-\omega_{n}}\left(\psi_{n}, i \omega_{n}^{2} \psi_{n}\right)\right\|^{2}<\infty .
$$

Theorem 2 is then applied again to conclude Theorem 7 .

\section{Concluding remarks}

By means of an abstract result on Riesz basis generation properties for discrete operators in general Hilbert spaces, we have shown that the usual Euler-Bernoulli beam equation with joint linear feedback is a Riesz system, which is one of the most profound properties for vibrating systems. Two further examples are presented to show that by this approach, the spectrum-determined growth condition, the asymptotic expression of eigenvalues as well as exponential stability can be readily obtained, giving a much clearer picture of the distribution of eigenvalues and greatly simplifies the proof by existing approaches. Moreover, our approach involves only some very elementary and straightforward 
calculations. This approach depends strongly on a better understanding of the asymptotic distribution of eigenvalues of the auxiliary free Riesz system. Unfortunately however, this is not always easy. A typical example can be found in Rebarber (1995) that still needs for further investigation.

Acknowledgements. This work was done during the first author's visit at the University of Hong Kong. The supports of the National Key Project of China and the Natural Science Foundation of China are greatly acknowledged.

\section{References}

[1] G. Chen, M.C. Delfour, A.M. Krall, and G. Payre, Modeling, stabilization and control of serially connected beam, SIAM J.Control \& Optim., 25(1987), 526-546.

[2] G. Chen et al. ., Analysis, design, and behavior of dissipative joints for coupled beams, SIAM J.Appl.Math., 49(1989), 1665-1693.

[3] G. Chen and J.X. Zhou, The wave propagation method for the analysis of boundary stabilization in vibrating structures, SIAM J.Appl.Math., 50(1990), 1254-1283.

[4] R. Rebarber, Exponential stability of coupled beams with dissipative joints: a frequency domain approach, SIAM J.Control \& Optim., 33(1995), $1-28$.

[5] F. Conrad and O. Morgul, On the stabilization of a flexible beam with a tip mass, SIAM J.Control \& Optim., 36(1998), 1962-1986.

[6] B.Z. Guo, The Riesz basis property of discrete operators and application to a Euler-Bernoulli beam equation with boundary linear feedback control, IMA J.of Math. Control \& Information, 2001a, in press.

[7] B.Z. Guo, Further result on a one-dimensional linear thermoelastic equation with Dirichlet-Dirichlet boundary condition, J. Australia Math. Soc. Ser. (B), 2001b in press.

[8] B.Z. Guo, Riesz basis approach to the stabilization of a flexible beam with a tip mass, SIAM J. Control \& Optim, 2001c, in press.

[9] G.H. Hardy and E.M. Wright, Introduction to the Theory of Numbers, 5th ed., Clarendon Press, Oxford, UK, 1979. 
[10] W.D. Zhu, Mote C.D. Jr and B.Z. Guo, Asymptotic distribution of eigenvalues of a constrained translating string, ASME J.of Applied Mechanics, (64)1997, 613-619.

[11] D. Henry, Linear autonomous neutral functional differential equations, Journal of Diff.Equs., 15(1974), 106-128.

[12] I. Singer, Bases in Banach Spaces I, Springer-Verlag, 1970.

Bao-Zhu Guo

Institute of Systems Science

Academy of Mathematics and System Sciences

Academia Sinica

Beijing 100081

China

E-mail: (bzguo@isso3.iss.ac.cn)

K.Y. Chan

Department of Mathematics The University of Kong Kong

Recibido: 9 de Junio de 2000 HONG KONG

Revisado: 19 de Julio de 200C 